\title{
ESTUDO ANATÔMICO DO XILEMA SECUNDÁRIO DE Mimosa trachycarpa Benth.
}

\author{
ANATOMY OF THE SECONDARY XYLEM OF Mimosa trachycarpa Benth.
}

\author{
José Newton Cardoso Marchiori ${ }^{1}$ Graciela Bolzon de Muñiz ${ }^{2}$
}

RESUMO

São descritos os caracteres microscópicos da madeira de Mimosa trachycarpa Benth., fornecidos dados quantitativos, determinações estereológicas e fotomicrografias de sua estrutura anatômica. Foram encontrados raios muito finos, parênquima paratraqueal, fibras libriformes não septadas, pontoações ornamentadas e poros agrupados em cachos, com arranjo tendente a diagonal. A estrutura da madeira é comparada com referências da literatura para a família Leguminosae e gênero Mimosa, em análise filogenética, taxonômica e ecológica.

Palavras-chave: anatomia da madeira, Leguminosae, Mimosa
trachycarpa, Stipellares. SUMMARY

The wood anatomy of Mimosa trachycarpa Benth. is described. Photomicrographs as well as quantitative and stereological data of its minute structure were furnished. Very fine rays, paratracheal parenchyma, libriform and not septated fibres, vestured pits and pores in clusters, tending to a diagonal pattern, were found. The wood anatomy was checked with literature of the Leguminosae family and genus Mimosa, considering its phylogenetical, taxonomical and ecological aspects.

Key words: wood anatomy, Leguminosae, Mimosa trachycarpa,Stipellares.

\section{INTRODUÇÃO}

O gênero Mimosa L. compreende mais de 400 espécies, que se distribuem pelas regiões quentes do continente americano, desde o sul dos Estados Unidos até o Uruguai e centro da Argentina.

A taxonomia das Mimosas baseia-se em BENTHAM (1875), que agrupou em duas secções, Habbasia e Eumimosa, cada uma contendo diversas séries botânicas. A secção Habbasia reúne as espécies com flores diplostêmones, ao passo que nas Eumimosae, as flores apresentam um número de estames igual ao de pétalas.

A espécie descrita no presente trabalho é um arbusto aculeado, densamente revestido de pêlos glanduloso-capitados. As folhas, com 2 a $5 \mathrm{~cm}$ de comprimento, compõem-se de 3 a 6 jugos e 9 a 12 pares de folíolos imbricados por pina, tendo estipelas cordadas e estípulas foliáceas. As flores são rosadas, octandras e dispostas em capítulos axilares pedunculados. Os frutos são pequenos e de poucas articulações (BURKART, 1979). A espécie encontra-se naturalmente em cerros e margens de cursos d'água, no Uruguai (LOMBARDO, 1964) e Rio Grande do Sul (BURKART, 1979).

\footnotetext{
${ }^{1}$ Engenheiro Florestal, Professor Titular, Dr., Departamento de Ciências Florestais, Centro de Ciências Rurais (CCR), Universidade Federal de Santa Maria (UFSM), 97119-900 - Santa Maria, RS. Autor para correspondência.

${ }^{2}$ Engenheiro Florestal, Professor Titular, Dr., Departamento de Engenharia e Tecnologias Rurais, Setor de Ciências Agrárias, Universidade Federal do Paraná, Curitiba, PR.
} 
Mimosa trachycarpa Benth. inclui-se na secção Habbasia, por suas flores diplostêmones, e na série Stipellares, por ser provida de estipelas foliáceas na base das pinas. Para a área de ocorrência natural de Mimosa trachycarpa são citadas outras 5 espécies da mesma série botânica: Mimosa amphigena Burk., Mimosa cruenta Benth., Mimosa hassleriana Chod., Mimosa uruguensis Hook. et Arn. (LOMBARDO, 1964) e Mimosa ostenii (BURKART, 1987).

Com relação à estrutura do xilema secundário, são poucas as referências encontradas na literatura sobre o gênero Mimosa e espécies da série Stipellares.

Em estudo de 11 espécies da Argentina, COZZO (1951) observou uma grande variação anatômica. Desta relação constavam Mimosa cruenta, Mimosa hassleriana, Mimosa ostenii e Mimosa uruguensis, pertencentes à série Stipellares. Na ausência de um traço comum a todo o grupo, o anatomista considerou-o como "estruturalmente heterogêneo".

Para Mimosa uruguensis foram referidos a predominância de poros em múltiplos e a presença de raios lenhosos geralmente unisseriados, compostos de células horizontais. A ocorrência de poros em arranjo dendrítico, foi também relatada para as outras 3 espécies da série Stipellares, examinadas pelo anatomista argentino.

Para Mimosa cruenta, MARCHIORI (1985) destacou a presença de uma porosidade dendrítica e sugeriu que o caráter tem valor taxonômico para o reconhecimento da série Stipellares. Foram também referidos a ocorrência de poros muito pequenos e extremamente numerosos, de elementos vasculares muito curtos, com placas de perfuração simples e providos de espessamentos espiralados tênues, de parênquima axial abundante, de raios fracamente heterogêneos, de fibras libriformes e a ausência de estratificação.

O xilema secundário de Mimosa trachycarpa é desconhecido, inexistindo referências sobre seus aspectos anatômicos. O presente trabalho visa estudar suas características microscópicas, contribuindo para o conhecimento taxonômico do gênero a que pertence.

\section{MATERIAIS E MÉTODOS}

O material estudado é procedente do Estado Rio Grande do Sul e consiste de 6 amostras de madeira, conservadas no Herbário e Xiloteca do Departamento de Ciências Florestais (HDCF), com os seguintes registros: HDCF 679. Pedreira do SOCEPE, Pinhal, Santa Maria. Marchiori, J.N.C., 10/81982; HDCF 685. Pinhal, Santa Maria, RS. Marchiori, J.N.C. 23/8/1982; HDCF 689. São Sepé, RS. Marchiori, J.N.C., 02/9/
1982; HDCF 700. Boqueirão, Santiago, RS. Marchiori, J.N.C., 17/9/82; HDCF 2800. Vila Etelvina, Santa Maria, RS. Longhi, S.J., $n^{\circ}$ 962, 20/11/1987; HDCF 2807. Cruz Alta, RS. Marchiori, J.N.C., n ${ }^{\circ} 427$, 18/8/1986.

De cada amostra de madeira foram preparados 3 corpos de prova, orientados para a obtenção de cortes anatômicos nos planos transversal, longitudinal radial e longitudinal tangencial. O amolecimento dos mesmos foi realizado por fervura em água. As secções anatômicas foram obtidas em micrótomo de deslizamento, regulado para a obtenção de cortes com a espessura nominal de $18 \mu \mathrm{m}$. Usou-se tripla-coloração, com Acridina-vermelha, Crisoidina e Azul-de-astra (DUJARDIN, 1964) e montagem de lâminas permanentes, com Entellan.

Para o preparo de lâminas de macerado, procedeu-se a dissociação do tecido lenhoso pelo método de Jeffrey (FREUND, 1970). Usou-se coloração com safranina e o mesmo meio de montagem anteriormente referido.

A terminologia adotada, as medições e descrição anatômica, seguiram as recomendações da COPANT (1973). Para a determinação da percentagem dos diferentes tipos celulares, usou-se a metodologia recomendada por MARCHIORI (1980). Os dados quantitativos e determinações estereológicas são apresentados na Tabela 1. As fotomicrografias foram tomadas em aparelho Carl Zeiss, no Laboratório de Anatomia da Madeira, da Universidade Federal do Paraná.

\section{DESCRIÇÃO DA MADEIRA}

Vasos: Muito numerosos, ocupando 18,7\% do volume da madeira. Porosidade difusa. (Figura 1.A). Poros de diâmetro pequeno (45 - $73-100 \mu \mathrm{m})$, de forma oval e paredes espessas, agrupados em cachos de numerosas unidades (Figuras 1.A; 2.A,B), com arranjo tendente a diagonal. Elementos vasculares muito curtos $(82-132-173 \mu \mathrm{m})$, com placas de perfuração simples e delicados espessamentos espiralados na parede celular. Apêndices geralmente ausentes ou então curtos (10 - $23-38 \mu \mathrm{m})$, em apenas uma das extremidades. Pontoações intervasculares alternas, poligonais, pequenas $(5-6-7,5 \mu \mathrm{m}) \mathrm{e}$ ornamentadas, com abertura inclusa, lenticular, tendente a horizontal (Figura 2.D). Pontoações raiovasculares muito pequenas até pequenas $(3-4,2-$ $6 \mu \mathrm{m}$ ), arredondadas e aparentemente simples (Figura 2.C). Pontoações parênquimo-vasculares muito pequenas até pequenas $(3,5-4,4-6 \mu \mathrm{m})$, semelhantes às raio-vasculares (Figura 2.C). 
Tahela I - Dados quantitativos e determinaçós estercológicas da madeira de Mimosa trachycarpa.

\begin{tabular}{|c|c|c|c|c|}
\hline CARACTLRÍSTICA & VALOR MÍNIMO & MÉDIA & VALOR MÁXIMO & $\begin{array}{l}\text { DESYIO } \\
\text { PADRĀO }\end{array}$ \\
\hline 1. Fraça de poros (\%) & 16,0 & 18,7 & 26,0 & 3,77 \\
\hline 2. Dismeiro tangencial de poros $(\mu \mathrm{m})$ & 45.0 & 73,0 & 100,0 & 13,12 \\
\hline 3. Comprimento clementos vasculares $(\mathrm{wm})$ & 82.0 & 132,0 & 173,0 & 20,94 \\
\hline 4. Comprimento de apéndices (..m) & 10.0 & 23.0 & 38,0 & 9.13 \\
\hline S. Diameun pontoaçdes intervasculares (im) & 5,0 & 6,0 & 7.5 & 0.69 \\
\hline 6. Diknetro puntusçōes raio-sastulares (um) & 3.0 & 4,2 & 6.0 & 0.75 \\
\hline 7. Diemerro pontosçes parénquimo-vasculares (6m) & 3.5 & 4,4 & 6,0 & 0.75 \\
\hline 8. Fraçto parènquina axial $(\%)$ & 10.0 & 11.8 & 16.0 & 2.40 \\
\hline 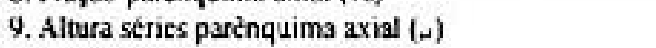 & 150.0 & 183,0 & 230,0 & 18.44 \\
\hline 10. Largura vélulas parènquima axial (wm) & 9.0 & 14,0 & 20,0 & 2,07 \\
\hline II. Altura cellulas fusiloumes (;-m) & 132.0 & 174,0 & 225,0 & 24,02 \\
\hline 12. Largurn oclulas fusiformes (..m) & 10.0 & 14,5 & 22.0 & 2.53 \\
\hline 13. Frapto tecido radial $(\%)$ & 8.0 & 10,0 & 12,0 & 1,67 \\
\hline 14. Frequência de raios (raiosínm) & 6.0 & 8.6 & 11,0 & 1.32 \\
\hline 15. Frapdu ruios unisseriadus (\%) & $1 \geq .0$ & 14,5 & 19,0 & 2,81 \\
\hline 16. Altura raios unisscriados $(. \mathrm{m})$ & 15.0 & 88,0 & 190,0 & 37,12 \\
\hline 17. Aitura raios unisocriadis & 1 & 7 & 14 & 2.97 \\
\hline 18 Largura raios unisscriados (um) & 6.0 & 10,0 & 14,0 & 1,86 \\
\hline 14. Fracto raios hisseriados $(\%)$ & 32.0 & 43,6 & 49.0 & 10,32 \\
\hline 20. Frą̧ao raios trisscriadns (\%) & 30.0 & 36.2 & 39.0 & 3,48 \\
\hline 21. Frapilo rraios tetrasseriados (\%) & 4,0 & 5.7 & 8.0 & 1.63 \\
\hline 22. Altura raios multixseriados (..m) & 1020 & 251.0 & 683,0 & 136.75 \\
\hline 23. Alturs raius multisseriados (celulis) & 8 & 19 & 54,0 & 10,78 \\
\hline 24. Largurn raios mulrisseriados (s m) & 150 & 24.0 & 34,0 & 8,64 \\
\hline 25. Largura raios multisseriados (ećlulas) & 2 & 3 & 4 & 0.71 \\
\hline 26. Fracto de fibras $(\%)$ & 53,0 & 59,5 & 66,0 & $\$ .39$ \\
\hline 27. Comprimento de fibras $(\mathrm{m})$ & $\$ 20,0$ & 602,0 & 780,0 & 36,72 \\
\hline 28. Diajmetro de fibras (am) & 10,0 & 15.0 & 20,0 & 2.62 \\
\hline 29. Diametro lume de fibras (um) & 2.5 & 6,6 & 11,5 & 2,45 \\
\hline 30. Espessura parede de fibras (um) & 2.5 & 4,3 & 5,6 & 0.69 \\
\hline
\end{tabular}

Parênquima axial: Pouco abundante, em disposição paratraqueal e ocupando $11,8 \%$ do volume da madeira. Em secção transversal, este parênquima constitui um envoltório nos agrupamentos racemiformes de poros (Figura 2.A,B), compondo eventualmente curtos segmentos tangenciais, ao estabelecer uma confluência entre os mesmos. Células de parênquima fusiformes com 132 - 174 - 225 $\mu$ m de comprimento por 10 - 14,5 - 22 $\mu \mathrm{m}$ de largura. Séries parenquimáticas axiais, com $150-183-230 \mu \mathrm{m}$ de

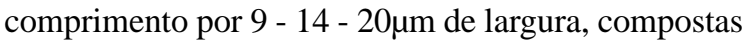
invariavelmente por apenas 2 células. Na transição do parênquima axial para o tecido fibroso não são raras as séries de cristais romboédricos, em 15 - 23 câmaras.

Raios: Numerosos (6 - 8,6 - 11/mm), compondo cerca de $10 \%$ do volume da madeira. Tecido radial homogêneo (Figura 1.B), composto apenas por células horizontais. Raios agregados, ausentes. Raios de relecionamento normal (Figura 1.C), freqüentemente fusionados em suas extremidades. Raios unisseriados abundantes (cerca de $14,5 \%)$, muito baixos (15 - $88-190 \mu \mathrm{m})$,

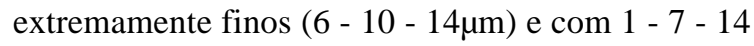
células de altura. Raios em sua maioria bisseriados $(43,6 \%)$, trisseriados $(36,2 \%)$ e, menos comumente, com 4 células de largura $(5,7 \%)$. Raios multisseriados

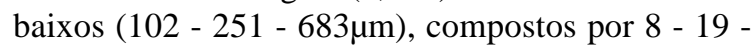
54 células de altura e muito finos (15 - $24-34 \mu \mathrm{m})$. Em plano tangencial, as células radiais tendem a ser isodiamétricas (Figura 1.C). Células envolventes, eretas, esclerosadas, latericuliformes, oleíferas, mucilaginosas e quadradas, ausentes na estrutura radial. Cristais romboédricos grandes e semelhantes aos do parênquima axial, pouco freqüentes e 

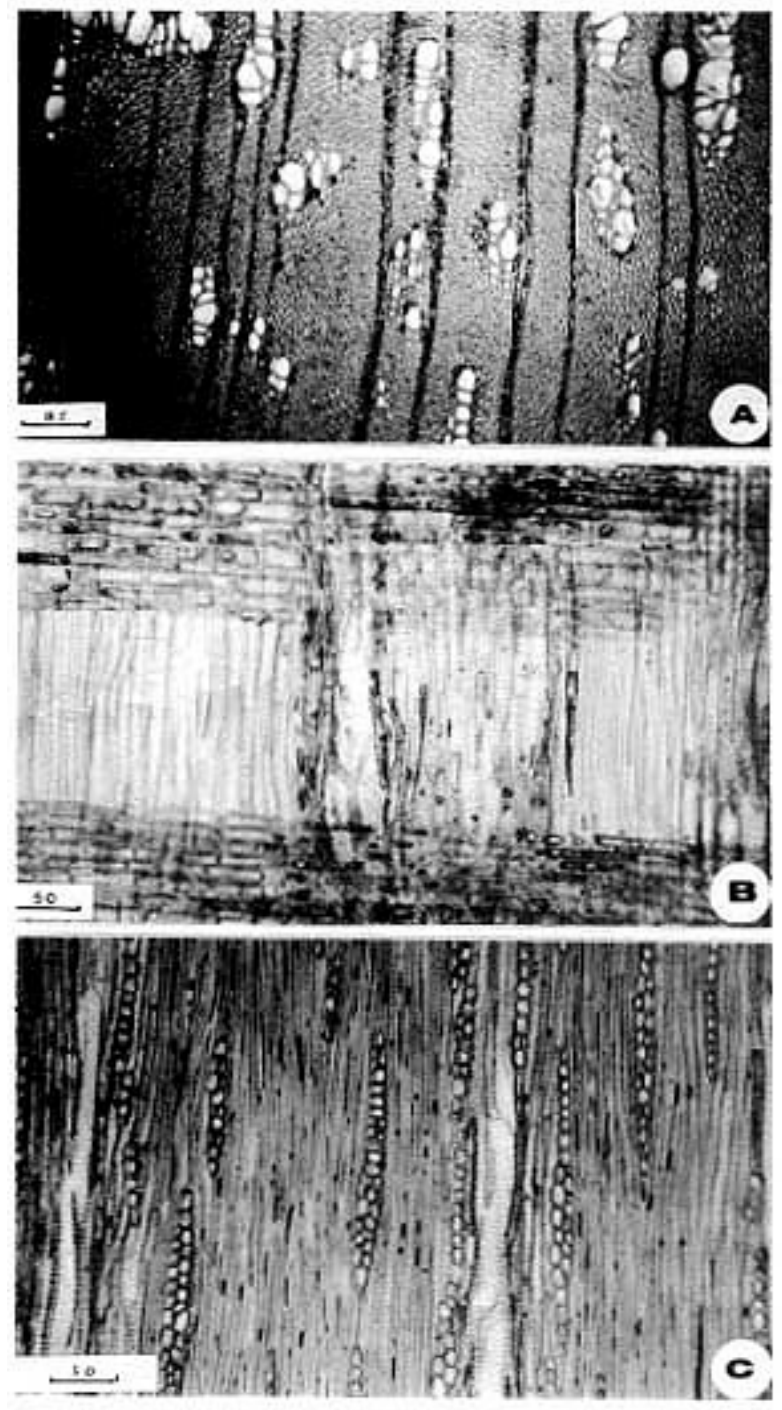

Figura 1 - Aspectos anatỏmicos da madeia de Mirnosa crachycarpa A. Corte transversal, mostrando um limite de anel de crescimento, porssidale difusa e puos sm ag upanentos raceniformes (em cxcho). d: nuanerosas unidades. B Cote Iengitudinal atalial, mosuando o curso de un vaso, ne cento da campo,e pare de dois reios homoginios, eompostos inteiranicnte de celulas horizortars. (C) Corte tongitudinal tangencial destacands raios nomais, firios (1-4-seriados) e com celulas te scesto isodiamétricu. (Todas as escalas en $، \mathrm{~m}$ ).

confinados às adjacências desta estrutura. Cristais romboédricos pequenos, abundantes em todas as células radiais.

Fibras: Ocupando 59,5\% do volume da madeira. Fibras libriformes, não septadas, freqüentemente gelatinosas, providas de diminutas pontuações simples, mais abundantes na face radial da parede. Fibras muito curtas (520 - $602-780 \mu \mathrm{m})$, estreitas $(10-15-20 \mu \mathrm{m})$ e de paredes delgadas a espessas $(2,5-4,3-5,6 \mu \mathrm{m})$.

Outros caracteres: Anéis de crescimento distintos (Figura 2.A,B). Canais secretores, tubos laticíferos e taniníferos, líber incluso e estratificação, ausentes.

\section{ANÁLISE DA ESTRUTURA ANATÔMICA}

Os principais caracteres anatômicos do xilema secundário de Mimosa trachycarpa são muito comuns em Leguminosas e atestam um alto grau de evolução. Destre estes, incluem-se elementos vasculares muito curtos, placas de perfuração simples, pontuações intervasculares ornamentadas, parênquima axial paratraqueal, raios homogêneos e fibras libriformes.

O agrupamento de poros em cachos, bem como a tendência dos mesmos ao arranjo diagonal, foram anteriormente registrados para Mimosa cruenta, Mimosa hassleriana, Mimosa ostenii e Mimosa uruguensis (COZZO, 1951), pertencentes à mesma série Stipellares e secção Habbasia. O autor argentino, todavia, não observou uma ligação taxonômica entre este grupo de espécies e o referido caráter anatômico. Esta correlação, observada inicialmente em Mimosa cruenta (MARCHIORI, 1985), é confirmada no presente trabalho, com o estudo da madeira de Mimosa trachycarpa Benth.

Cabe também destacar que a espécie em estudo também apresenta raios muito finos e espessamentos espiralados tênues em vasos, à semelhança de Mimosa cruenta. Para a confirmação de um eventual significado taxonômico dos mesmos para a série Stipellares Benth., torna-se todavia necessária uma investigação mais ampla no grupo, abrangendo um maior número de espécies.

A presença de poros de diâmetro muito pequeno e agrupados em cachos de numerosas unidades, sugere um valor adaptativo a ambientes secos, como os ocupados preferentemente pela espécie em estudo. Para a identificação de Mimosa trachycarpa destacam-se, ainda, a ausência de septos em fibras e a presença de cristais nos parênquimas axial e radial.

\section{CONCLUSÕES}

A estrutura anatômica da madeira de Mimosa trachycarpa permite a formulação das seguintes conclusões:

- A madeira apresenta uma estrutura anatômica evoluída, à semelhança das demais leguminosas. 

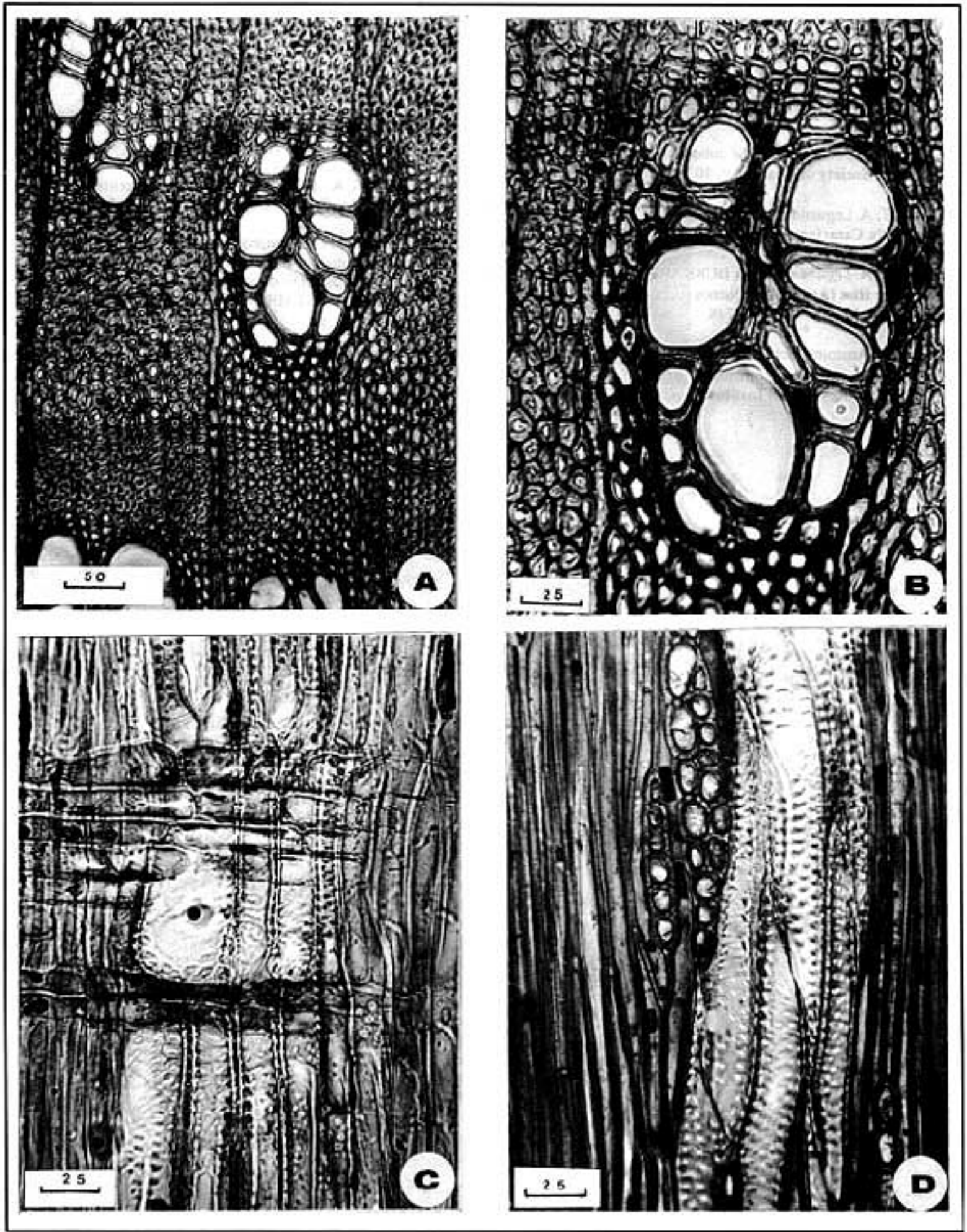

Figurs 2 - Detalhes anotemicos dz madeira d: Minesa irachycarpa, A. Corte tranyversal, destacando o linite de um ancl de crescincnto c cachos de poros $\mathrm{cm}$ cashos, rodeados de parinquina axial. B Mesnos aspectos da fotogafia anterior, mis con maior aumento C. Aspecio longirudinal rad al da madeira, mostrando ralos homogencos e pontuą̧es parénquimo-vasculares e ralc-vasculares urredondadas, de tamarho muito pequens a pequeno. D. Aspecto de um corte longitudinal tangencial, mostrando raioss fincs (uni c bisstriados) $\mathrm{e}$ um teike de vasos eom pontuaçies intervasculares em aranje alterio. (Todas as escalas $\mathrm{cm} \mu \mathrm{m}$ ).

Ciência Rural, v. 27, n. 2, 1997. 
- A presença de poros agrupados em cachos e com arranjo tendente à diagonal, são característicos das mimosas da série Stipellares (secção Habbasia).

- A ocorrência de raios muito finos e espessamentos espiralados em vasos, são sugestivos do mesmo grupo taxonômico, mas requer uma posterior confirmação, mediante estudo mais abrangente na referida série botânica.

A presença de poros muito pequenos e agrupados, tem valor adaptativo à xeromorfia.

\section{REFERÊNCIAS BIBLIOGRÁFICAS}

COPANT - COMISSÃO PANAMERICANA DE NORMAS TÉCNICAS. Descrição geral, macroscópica e microscópica da Madeira. Esquema I de remendação. Colômbia, 1973. 19 p. (COPANT 30).

BENTHAM, G. Revision of the suborder Mimoseae. Transact Linnean Society of London, v. 30, p. 335-664, 1875.

BURKART, A. Leguminosas Mimosoídeas. In: REITZ, P.R. Flora Ilustrada Catarinense. Itajaí, 1979. 299 p.
BURKART, A. Leguminosae. In: BURKART, A. Flora Ilustrada de Entre Rios (Argentina). Buenos Aires: Coleccion Cientifica del INTA, 1987. v. 6, p. 442-738.

COZZO, D. Anatomia del Leño Secundario de las Leguminosas Mimosoideas y Caesalpinoideas Argentinas Silvestres y Cultivadas. Revista del Instituto Nacional de Investigacion de las Ciencias Naturales, Buenos Aires, v. 2, n. 2, p. 63-146, 1951.

DUJARDIN, E.P. Eine neue holz-zellulosenferbung. Mikrokosmos, n. 53, p. 94, 1964.

FREUND, H. Handbuch der Mikroskopie in der Technik. Frankfurt, Umsham Verlag, 1970. 379 p.

LOMBARDO, A. Flora Arborea y Arborescente del Uruguay. Montevideo, Concejo Departamental, 1964. 151 p.

MARCHIORI, J.N.C. Comprovação da viabilidade de utilização da secção longitudinal tangencial para a determinação histométrica dos elementos axiais do xilema secundário. In: CONGRESSO FLORESTALESTADUAL, IV. Anais do. Nova Prata, 1980. p. 180-184.

MARCHIORI, J.N.C. Anatomia da madeira de Mimosa cruenta Benth. (Leguminosae Mimosoideae). Ciência e Natura, Santa Maria, v. 7, p. 73-81, 1985. 